\section{JTI}

JOURNAL OF

TRAUMA AND INJURY

\title{
Unplanned Reoperation Rate at a Government-Designated Regional Trauma Center in Gangwon Province
}

\author{
Minju Kim, M.D., Seongyup Kim, M.D. \\ Department of Surgery, Wonju Severance Christian Hospital, Wonju, Korea
}

Received: July 24, 2020

Revised: October 5, 2020

Accepted: October 22, 2020

\section{Correspondence to}

Seongyup Kim, M.D.

Department of Surgery, Wonju Severance Christian Hospital, Regional Trauma

Center, Yonsei University Wonju College

of Medicine, 20 Ilsan-ro, Wonju 26426,

Korea

Tel: $+82-33-741-0573$

Fax: +82-33-741-0574

E-mail: sykimvs@yonsei.ac.kr

Purpose: Determining appropriate ways to assess health care quality within the National Health Insurance System is of interest to both the Korean government and the medical community. However, in the trauma field, the number of indicators used to evaluate surgical quality is limited. Using data collected over 5 years at Wonju Severance Christian Hospital Trauma Center in Korea, this study aimed to determine whether the unplanned reoperation rate in the field of trauma surgery could be used to assess the quality of an institution's surgical care.

Methods: In total, 665 general surgical procedures were performed at the Trauma Center in 453 patients with abdominopelvic injuries from January 2015 to December 2019. Data were collected from the Trauma Center's data registry and medical records, and included information regarding patients' demographic characteristics, the type of index operation, and the reason for unplanned reoperations.

Results: A total of 453 index operations were evaluated. The proportion of patients with an Injury Severity Score (ISS) $>15$ was $48-70 \%$ over the 5 -year period, with an unplanned reoperation rate of 2.1-9.3\%. Patients had an average ISS score of 17.5, while the average Abbreviated Injury Scale Score was 2.87. Unplanned reoperations were required in about $7 \%$ of patients. The most common complications requiring reoperation were recurrent bleeding (26.9\%), wound problems (26.9\%), intestinal infarction (15.4\%), and anastomosis site leakage (7.7\%). The procedures most frequently requiring unplanned reoperations were bowel surgery (segmental resection, primary repair, enterostomy, etc.) $(24.5 \%)$ and preperitoneal pelvic packing (10.6\%).

Conclusions: The proportion of reoperations was confirmed to be affected by injury severity.

Keywords: Wounds and injuries; Reoperation; Quality of health care

This is an Open Access article distributed under the terms of the Creative Commons Attribution Non-Commercial License (http://creativecommons.org/licenses/by-nc/4.0/) which permits unrestricted noncommercial use, distribution, and reproduction in any medium, provided the original work is properly cited. 


\section{INTRODUCTION}

The mortality rate, length of stay in the intensive care unit or hospital, and complication rate are often used to evaluate individual institutions' quality of surgical care [1]. These metrics have also been used by the Health Insurance Review and Evaluation Commission to assess the appropriateness of medical services in the context of the expansion of the new Diagnosis-Related Group system in Korea [2]. However, focusing on the mortality rate to evaluate an institution's surgical quality may not be helpful, as the mortality rate does not necessarily reflect the problems that occur in the course of general patient care [3]. The length of hospitalization in the intensive care unit and in-hospital days may also vary depending on the country and region in which a medical institution is located, as well as the medical and economic conditions; thus, it is challenging to accurately evaluate the surgical quality of an institution using these parameters [4,5].

Surgical complications occur along a broad spectrum, depending on the definition. If the details of each complication are not accurately defined and classified, the accuracy of surgical quality evaluation may be reduced. As unavoidable and unplanned reoperations are required in the event of a number of common surgical complications, the rate of unplanned reoperations can be evaluated fairly accurately using a data registry in a retrospective manner [6-9]. Accordingly, the unplanned reoperation rate can be used as a marker for evaluating an institution's surgical quality.

This study aimed to investigate the unplanned reoperation rate and general surgery characteristics at Wonju Severance Christian Hospital Trauma Center, a government-designated regional Trauma Center in Gangwon Province, Korea.

\section{METHODS}

We analyzed Wonju Severance Christian Hospital Trauma Center's data registry from January 2015, its opening year, to December 2019. General surgical procedures requiring general anesthesia were reviewed to investigate the unplanned reoperation rate and the clinical character- istics of patients who underwent unplanned reoperations.

The index operation was defined as the first general surgery performed in trauma patients who presented to Wonju Severance Christian Hospital Trauma Center for the first time after an accident. We defined an unplanned reoperation as any surgical procedure required due to a complication related to the index operation [6]. If multiple operations were performed in a patient, the surgical records were reviewed to prioritize the procedure performed for damage that significantly impacted vital signs.

In principle, the institutional guidelines state that gauze must be removed 24-48 hours after operations involving preperitoneal pelvic packing or intra-abdominal packing. For cases in which packing removal was not performed within this time frame or re-packing surgery was performed, we reviewed medical records to determine why this was done. If the reason was related to uncontrollable hemorrhage, we regarded the second operation as an unplanned reoperation. In cases of temporary abdominal closure, we reviewed medical records to identify the indications for subsequent operations. We considered a subsequent operation to be unplanned reoperation if the indications were related to either definite hemorrhage or bowel content leakage.

Table 1. Characteristics of index general surgery cases

\begin{tabular}{|lc|}
\hline Total operation & 655 \\
\hline Index operation in abdominopelvic trauma patient & 453 \\
Male & $316(69.8)$ \\
Female & $137(30.2)$ \\
Mean age (years) & 52.5 \\
Injury mechanism & \\
Driver \& passenger TA & $205(45.2)$ \\
Stab injury & $83(18.3)$ \\
Pedestrian TA & $36(7.9)$ \\
Motorcycle TA & $36(7.9)$ \\
Fall down & $34(7.5)$ \\
Blunt trauma except TA & $31(6.8)$ \\
Slip down & $13(2.9)$ \\
Injury by machine & $11(2.4)$ \\
Unknown & $4(0.8)$ \\
\hline
\end{tabular}

Values are presented as number (\%).

TA: traffic accident. 


\section{Statistical analysis}

The data were collected by two general surgeons and validated based on expert judgment. We used the chi-square test and the Student $t$-test to analyze the correlations between variables. The chi-square test and linear-by-linear associations were used to analyze trends in Injury Severity Scores (ISS) and unplanned reoperations by year. A $p$-value $<0.05$ was considered to indicate statistical significance when calculating the corresponding $95 \%$ confidence intervals. All analyses were performed using SPSS version 24.0 (IBM Corp., Armonk, NY, USA).

\section{RESULTS}

A total of 655 general surgical procedures were performed at the Trauma Center in 453 patients with Abbreviated Injury Scale (AIS) scores from 1 to 4 . Driver and passenger accidents were the most common injury mechanism

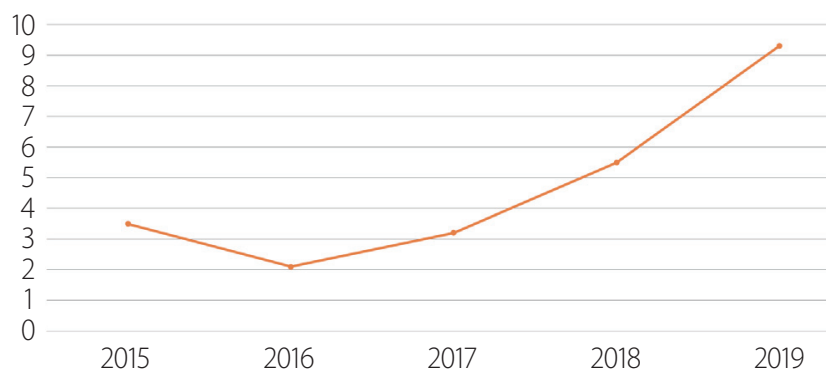

Fig. 1. Trend in the unplanned reoperation rate (\%). in patients who underwent general surgery. The characteristics of patients who underwent general surgery are described in Table 1.

No significant trend was found in the proportion of cases involving an ISS $>15$ and unplanned reoperations ( $p=0.295, p=0.718$ ) (Figs. 1, 2). The most frequent index operation type that required an unplanned reoperation was bowel surgery (Table 2). Bleeding and wound problems were the most common cause of unplanned reoperations, followed by intestinal infarction. There were two malpractice cases. In one case, aortobifemoral bypass was performed on the right side; common iliac artery rupture occurred after the patient slipped and fell, and graft revision was performed to correct malpositioning of the graft. In the other case, preperitoneal pelvic packing was done for a pelvic fracture, but colostomy formation was eventually performed for a neglected rectal injury (Table 3).

The ISS scores were significantly higher in patients who underwent unplanned reoperations than in those who did

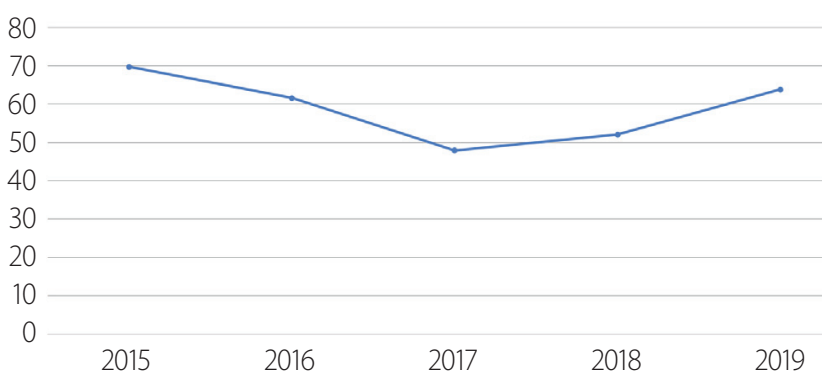

Fig. 2. Trend in the proportion (\%) of patients with an ISS >15.

Table 2. Types of index operations and unplanned reoperation rate

\begin{tabular}{|lcc|}
\hline Procedure & Index operation & Unplannded reoperation \\
\hline Bowel surgery (segmental resection, primary repair, enterostomy etc.) & 71 & $12(16.9)$ \\
\hline Preperitoneal pelvic packing & 47 & $3(6.4)$ \\
Solid organ bleeding control & 146 & $8(5.4)$ \\
Vascular bleeding control & 29 & $1(3.4)$ \\
Mesenteric bleeding control & 132 & $2(1.5)$ \\
Abdominal wall repair & 11 & $0(0)$ \\
Explorative laparotomy only & 14 & $0(0)$ \\
Others & & 0 \\
Total & 3 & $2(0)$ \\
\hline
\end{tabular}

Values are presented as number (\%).

aTwo cases of omental bleeding and one case of diaphragm rupture. 
not require reoperation $(p=0.003)$. The average AIS score in the abdomen was also significantly higher in patients who underwent unplanned reoperations $(p=0.005)$. A significant correlation between hospital length of stay and reoperation was also found (Table 4).

\section{DISCUSSION}

Few studies have been conducted on the unplanned reop-

Table 3. Causes of unplanned reoperations

\begin{tabular}{|lc|}
\hline Cause & \\
\hline Bleeding & $7(26.9)$ \\
Wound problem & $7(26.9)$ \\
Intestinal infarction & $4(15.4)$ \\
Sepsis & $3(11.5)$ \\
Anastomosis site leakage & $2(7.7)$ \\
Malpractice & $2(7.7)$ \\
lleus & $1(3.8)$ \\
Total & $26(100)$ \\
\hline
\end{tabular}

Values are presented as number (\%). eration rate in the field of trauma surgery. According to a study conducted by University Medical Center Utrecht in 2019 , about $21 \%$ of patients undergoing abdominal trauma surgery underwent unplanned abdominal reoperations [10]; another level 1 Trauma Center reported a rate of roughly $20 \%$ [11]. A study conducted on unplanned abdominal reoperations after abdominal trauma surgery in the U.S. in 1978 [12] and a Russian study in 1985 [13] showed unplanned reoperation rates of $8.9 \%$ and $10.6 \%$, respectively. Considering the results of the aforementioned U.S., Russian, and Dutch studies, the overall rate of unplanned reoperations for abdominal trauma surgery can be estimated to be around $10-20 \%$.

In this study, the unplanned reoperation rate for trauma surgery in the general surgery field was 5.2\% (35 of 665), the average ISS was 17.5, and the average abdominal AIS score was 2.87. Wonju Severance Christian Hospital Trauma Center's reoperation frequency was lower than that reported in the University Medical Center Utrecht 2019 study (mean ISS, 27.5); this is attributed to the relatively low ISS and abdominal AIS scores at Wonju Severance Christian Hospital Trauma Center, although there were similarities in both the indications for unplanned

Table 4. Comparisons between patients who did and did not undergo unplanned reoperations

\begin{tabular}{|lccc|}
\hline & $\begin{array}{c}\text { Unplanned reoperation } \\
\text { group (n=26) }\end{array}$ & $\begin{array}{c}\text { No-unplannded reoperation } \\
\text { group ( } \mathbf{n = 4 2 7})\end{array}$ & p-value \\
\hline Age (mean) & 55 & 52.1 & 0.387 \\
ISS score (mean) & 21.92 & 17.28 & 0.003 \\
Average abdominal AIS grade (mean) & 3.38 & 2.85 & 0.005 \\
Hospital length (mean) & 60.23 & 24.22 & 0.000 \\
Mortality rate & 0.27 & 0.15 & 0.096 \\
Injury mechanism (\%) & & & 0.062 \\
Driver \& passenger TA & $8 / 205(3.9)$ & $197 / 205(96.1)$ & \\
Stab injury & $2 / 83(2.4)$ & $81 / 83(97.6)$ & \\
Pedestrian TA & $4 / 36(11.1)$ & $32 / 36(88.9)$ & \\
Motorcycle TA & $6 / 36(16.7)$ & $30 / 36(83.3)$ & \\
Fall down & $2 / 34(5.9)$ & $32 / 34(94.1)$ & $29 / 31(93.5)$ \\
Blunt trauma except TA & $2 / 31(6.5)$ & $11 / 11(100)$ & \\
Injury by machine & $0 / 11(0)$ & $11 / 13(84.6)$ & \\
Slip down & $2 / 13(15.4)$ & $4 / 4(100)$ & \\
Unknown & $0 / 4(0)$ & & \\
\hline
\end{tabular}


reoperations and the injury mechanism with respect to the index operation.

We performed this pilot study for the prospective quality management of general surgery at the Wonju Severance Christian Hospital Trauma Center. Initially, we assumed that the unplanned reoperation rate would decrease over time after 2015, the year the Trauma Center opened. However, there was no significant trend in the unplanned reoperation rate over time. A tendency for the unplanned reoperation rate to increase was observed, but it was not statistically significant. One of the major reasons for this finding may have been the influx of junior surgeons with little clinical experience when the Trauma Center was established. Therefore, improvement is anticipated in the future.

\section{Limitations}

The finding of a lower rate of unplanned reoperations in our study than in other abdominal trauma studies could be due to several reasons. First, our study did not include cases in which the initial treatment plan was conservative management; thus, cases involving the failure of nonoperative management were excluded. Second, the data were extracted through a retrospective review of medical records, not from a complications registry. Some problems may occur in the collection of retrospective data. For an accurate analysis of overall surgical quality, further studies involving prospective data collection are needed.

\section{CONCLUSION}

The reoperation rate was confirmed to be affected by injury severity. Although statistical significance was not found, it is reasonable to posit that surgeons' skills may affect the proportion of reoperations.

\section{REFERENCES}

1. Scott JW, Olufajo OA, Brat GA, Rose JA, Zogg CK, Haider AH, et al. Use of national burden to define operative emergency gen- eral surgery. JAMA Surg 2016;151:e160480.

2. Jeon MJ, Choo SP, Kwak YH, Kim DW, Kim EH. The effect of diagnosis-related group payment system on the quality of medical care for pelvic organ prolapse in Korean tertiary hospitals. PLoS One 2019;14:e0220895.

3. Ghaferi AA, Birkmeyer JD, Dimick JB. Variation in hospital mortality associated with inpatient surgery. N Engl J Med 2009;361:1368-75.

4. Achanta A, Nordestgaard A, Kongkaewpaisan N, Han K, Mendoza A, Saillant N, et al. Most of the variation in length of stay in emergency general surgery is not related to clinical factors of patient care. J Trauma Acute Care Surg 2019;87:408-12.

5. Ramsay G, Wohlgemut JM, Jansen JO. Emergency general surgery in the United Kingdom: a lot of general, not many emergencies, and not much surgery. J Trauma Acute Care Surg 2018;85:500-6.

6. Kroon HM, Breslau PJ, Lardenoye JW. Can the incidence of unplanned reoperations be used as an indicator of quality of care in surgery? Am J Med Qual 2007;22:198-202.

7. Ploeg AJ, Lange CP, Lardenoye JW, Breslau PJ. The incidence of unplanned returns to the operating room after peripheral arterial bypass surgery and its value as indicator of quality of care. Vasc Endovascular Surg 2008;42:19-24.

8. Birkmeyer JD, Hamby LS, Birkmeyer CM, Decker MV, Karon NM, Dow RW. Is unplanned return to the operating room a useful quality indicator in general surgery? Arch Surg 2001;136:405-11.

9. Fröschl U, Sengstbratl M, Huber J, Függer R. Unplanned reoperations for infection complications: a survey for quality control. Surg Infect (Larchmt) 2006;7:263-8.

10. Hietbrink F, Smeeing D, Karhof S, Jonkers HF, Houwert M, van Wessem K, et al. Outcome of trauma-related emergency laparotomies, in an era of far-reaching specialization. World J Emerg Surg 2019;14:40.

11. Scriba MF, Laing GL, Bruce JL, Clarke DL. Repeat laparotomy in a developing world tertiary level surgical service. Am J Surg 2015;210:755-8.

12. Driver T, Kelly GL, Eiseman B. Reoperation after abdominal trauma. Am J Surg 1978;135:747-50.

13. Iunko MA, Iurmin EA, Labaĭ EN. Relaparotomy in abdominal injuries. Vestn Khir Im I I Grek 1985;134:94-8. 